\title{
MODELING CORE FLUID MOTTONS AND THE DRIFT OF MAGNETIC FIELD PATTERNS AT THE CMB BY USE OF TOPOGRAPHY OBTAINED BY SEISMIC INVERSION
}

\author{
Monica D. Kohler \\ Seismological Lab, California Institute of Technology
}

David. J. Stevenson

Division of Geological and Planetary Sciences, California Institute of Technology

\begin{abstract}
The thermal wind equations, in which the Coriolis force is balanced by pressure gradients and horizontal density gradients rather than by Lorentz forces, are used to describe patterns of magnetic field drift associated with core fluid motions near the core-mantle boundary (CMB). The advection of magnetic field may be due in part to the flow driven by such horizontal temperature gradients, just as EastWest air flow is driven by North-South temperature gradients in the Earth's atmosphere. It is argued that this flow may be concentrated in a shell near the CMB, and the horizontal temperature gradients are expected to be directly proportional to horizontal gradients in CMB topography, the lowest harmonics of which are approximately constrained in seismology. Part of the zonal drift is then associated with the $\mathrm{l}=2, \mathrm{~m}=0$ harmonic of $\mathrm{CMB}$ topography, and anticyclones are attached to topographic highs (thermal highs). Comparison of our derived flow pattern with those determined purely by magnetic field observations provides tentative support for our model.
\end{abstract}

\section{Introduction}

The relationship between the Earth's mantle and core is the focus of numerous studies which consider the thermal and morphological nature of the core-mantle boundary (CMB) and its influence on core fluid motions. Various types of coremantle interactions have been proposed by which the morphology of the CMB, and the dynamics and temperature variations in the lower mantle affect motions of outer core fluid. Hide (1969) proposed a coupling mechanism which involved a hydrodynamical interaction between core fluid motions and undulations in CMB topography. Jones (1977) proposed that thermal interaction influenced field geometry and caused some geomagnetic properties (e.g., reversal frequency) to vary on a mantle convection timescale. In their secular variation study, Bloxham \& Gubbins (1985) suggested thermal, electromagnetic, and topographic coremantle interactions to explain the existence of static features in the Earth's magnetic field. The relationship was explored further by Bloxham \& Gubbins (1987) who proposed thermal interaction between the core and lower mantle, where large lateral temperature variations just above the CMB influenced convection in the core. Core-mantle interaction has also been invoked to explain certain features of secular variation in the Earth's magnetic field such as westward drift. Such

Copyright 1990 by the American Geophysical Union.

Paper number 90GL01479

$0094-8276 / 90 / 90 G L-01479 \$ 03.00$ nondipole features are believed by some to be the result of differential rotation of outer core fluid relative to the deep outer core. In addition to using core-mantle interaction to explain stationary features of secular variation (Gubbins \& Richards, 1986), Olson (1989) maintained that azimuthal drift of core fluid could be partially accounted for by thermal wind flow and that lateral temperature differences as small as $10^{-3} \mathrm{~K} / \mathrm{km}$ could significantly excite thermal winds.

\section{The Model}

We develop a simple model for part of the core fluid motion due to core-mantle boundary topography. The basic idea is as follows: relatively cold mantle just above the CMB is more dense and will sink causing a depression in the CMB, whereas relatively hot mantle is less dense and will rise, resulting in positive CMB topographic relief or "bumps". The relationship between the deformation of an interface and the pattern of convective thermal anomalies is not necessarily so simple [cf. Hager and Clayton, 1989] especially if the lowermost mantle (D") is a compositional layer [Lay, 1989] but we have chosen the most commonly assumed correlation. Cold, topographic lows cool the core from above and promote more vigorous vertical convection in the locally cold core fluid. Warm, topographic highs are associated with lower vertical heat flow and warmer core fluid. This is illustrated in Figure 1. Simple mixing length recipes for turbulent convection suggest temperature deviations away from an adiabat of order $10^{-3} \mathrm{~K}$ but with a large uncertainty [Stevenson, 1987]. These will also be the horizontal temperature variations on an equipotential. Horizontal as well as vertical heat flows can be expected [Flasar and Gierasch, 1978] and the relationship between the magninude of the mantle temperature fluctuations and those in the core may well be non-linear, but the sense of the correlation is clear: topographic highs of the CMB are associated with thermal highs in the outermost core.

In our consideration of the dynamics, we adopt the standard approach in which fluid acceleration relative to the rotating frame is ignored and viscous effects are ignored. We also neglect the Lorentz force in the outermost core because the toroidal component of the field should decrease to a low value in the outermost few hundred kilometers of the core due to the low conductivity of the mantle [Merrill and McElhinny, 1983]. The Lorentz force is dominated by a term that is proportional to the cross product of this toroidal field with the poloidal current. Here, we exclude the artificiality of current sheets that arise in some dynamo models. We also adopt the Boussinesq approximation in which the only dynamically significant density variations are those caused by thermal 


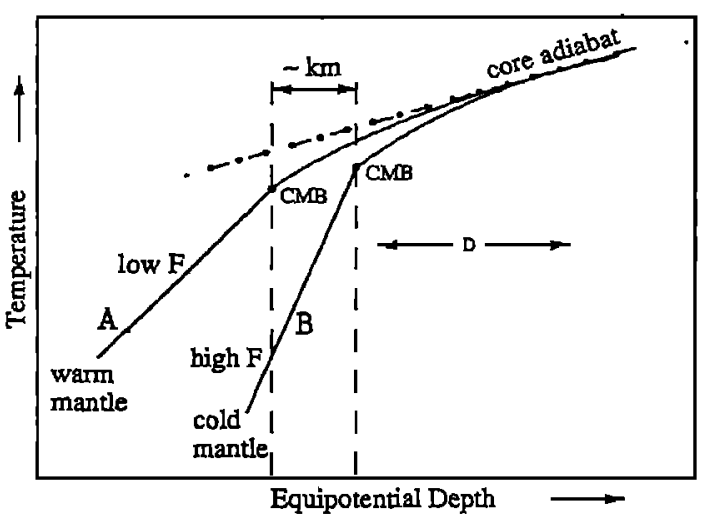

Fig. 1. Schematic representation of temperature profiles near $\mathrm{CMB}$. Temperature and depth scales are shown grossly distorted to clarify the important features. Profile A corresponds to a low heat flow region and topographic high. Profile $B$ is a high heat flow region and topographic low. Notice that at a given depth within the core, the temperature on profile $A$ is high relative to profile $B$. This correlation of topographic and thermal highs is central to the model described in the text. Note also that the absolute temperature at the CMB is lower for the high heat flow case (A) than for the low heat flow case (B), because the $\mathrm{CMB}$ on profile $\mathrm{A}$ is at a lower pressure. This effect is actually much bigger than the temperature difference at a fixed equipotential depth, but dynamically irrelevant.

expansion. We then arrive at the standard thermal wind equation, well known to atmospheric dynamicists [e.g., Pedlovsky, 1979] and occasionally discussed for the core [e.g., Olson, 1989]:

$$
2 \vec{\Omega} \times \overrightarrow{\mathrm{v}}=-\frac{\vec{\nabla} \mathrm{P}^{\prime}}{\rho_{\mathrm{O}}}-\overrightarrow{\mathrm{g}} \alpha \mathrm{T}
$$

where $\vec{\Omega}$ is the mean Earth angular velocity, $\vec{v}$ is the fluid velocity relative to this frame, $\mathrm{P}^{\prime}$ is the non-hydrostatic component of the fluid pressure, $p_{0}$ is the mean fluid density, $\alpha$ is the coefficient of thermal expansion, $T$ is the deviation of the temperature away from some mean core adiabat and $\vec{g}$ is the gravitational acceleration. The solution to the equation of motion can be found by taking the curl of both sides; however, by doing so, information concerning any flow that is purely geostrophic (i.e., Coriolis force balanced by a pressure gradient) is lost. We get

$$
(2 \vec{\Omega} \cdot \vec{\nabla}) \vec{v}=\vec{\nabla} \times(\overrightarrow{\mathrm{g}} \alpha \mathrm{T})
$$

We now make the further assumption that the thermal wind is primarily confined to a layer that is thin compared to the core radius. This is not a boundary layer (which is much thinner yet) but a layer in which the horizontal temperature gradients and the associated wind are dynamically dominant. This layer could be of order one hundred kilometers thick (but with a large uncertainty) and arise in two ways. First, it could be the region within which the boundary-generated buoyancy driving thermal convection is confined. This is in the spirit of local prescriptions for turbulent convection [Monin and Yaglom, 1971], if one assumes that the mixing length is equal to the distance from the CMB. According to this view, the temperature anomaly $\mathrm{T}$ decays in amplitude over some characteristic distance $D \ll R_{c}$, the core radius, as illustrated in Figure 1. The second possible reason for this thin layer approximation is that it may correspond to the region in which the Lorentz force is small. At deeper levels, there is a large enough toroidal field that flows of the type we consider are effectively damped. In either case we must seek solutions to equation (2) in which the velocity decays over a characteristic distance $\mathrm{D}$ as one goes downward into the core. These solutions have the property that the shear is parallel to the flow, i.e.,

$$
\frac{\partial}{\partial} \rightarrow \frac{1}{D}
$$

Since $D \ll R_{c}$, it follows that $\theta$ and $\phi$ derivatives of the velocity (but not the temperature) can be neglected relative to radial derivatives. (This is exactly analogous to the meteorological approach to thermal winds.) We then have

$$
\begin{gathered}
\mathrm{v}_{\theta}=-\frac{\mathrm{g} \alpha \varepsilon}{2 \Omega \sin \theta \cos \theta} \frac{\partial \mathrm{T}}{\partial \phi} \\
\mathrm{v}_{\phi}=\frac{\mathrm{g} \alpha \varepsilon}{2 \Omega \cos \theta} \frac{\partial \mathrm{T}}{\partial \theta}
\end{gathered}
$$

for the values of these velocity components at the CMB, where $\varepsilon=\frac{D}{R}$. As usual, $\theta$ is the colatitude, $\phi$ is longitude, and $\mathrm{g}=\mid \overrightarrow{\mathrm{g}} !$.

Continuity dictates that $v_{I}$ is smaller than $v_{\theta}$ or $v_{\phi}$ by a factor of $\varepsilon \ll 1$. Notice, however, that the predicted flow is not purely toroidal in general, although toroidal components tend to dominate. In the limit of fairly rapid $\theta$-variation of $T$ not near the equator (i.e., $\frac{T}{\varepsilon} \gg\left|\frac{\partial T}{\partial \theta}\right| \gg T ; \theta \neq \frac{\pi}{2}$ ) it is clear that the vorticity of the flow is radial and proportional to $\nabla^{2} T$ with a positive (negative) constant of proportionality in the Northern (Southern) hemisphere. This means that anticyclones are associated with temperature highs, the same as meteorological experience.

Quantitatively, the fundamental assumption of our model is the expression that core temperature variations are proportional to $\mathrm{CMB}$ topography variations, or

$$
T(\theta, \phi)=\beta \mathrm{f}(\theta, \phi)
$$

where $\beta$ is the constant of proportionality, and $T$ and $f$ are assumed to have zero means. If CMB topography is given in terms of a spherical harmonic expansion, the temperature is expressed in terms of the same expansion coefficients multiplied by $\beta$ :

$$
\begin{array}{r}
\mathrm{T}(\theta, \phi)=\beta \sum_{\ell=0}^{\mathrm{L}} \sum_{\mathrm{m}=0}^{\ell} \mathrm{P}_{\ell}^{\mathrm{m}}(\cos \theta)\left[\mathrm{g}_{\ell}^{\mathrm{m}} \cos \mathrm{m} \phi+\mathrm{h}_{\ell}^{\mathrm{m}} \sin \mathrm{m} \phi\right](6) \\
\mathrm{f}(\theta, \phi)=\sum_{\ell=0}^{L} \sum_{\mathrm{m}=0}^{\ell} \mathrm{P}_{\ell}^{\mathrm{m}}(\cos \theta)\left[\mathrm{g}_{\ell}^{\mathrm{m}} \cos \mathrm{m} \phi+\mathrm{h}_{\ell}^{\mathrm{m}} \sin \mathrm{m} \phi\right](7)
\end{array}
$$

We cannot justify rigorously this choice. However, the numerical values are plausible. For $\beta=10^{-3} \mathrm{~K} / \mathrm{km}$, and topographic relief $\sim \mathrm{km}$, we have temperature fluctuations not 

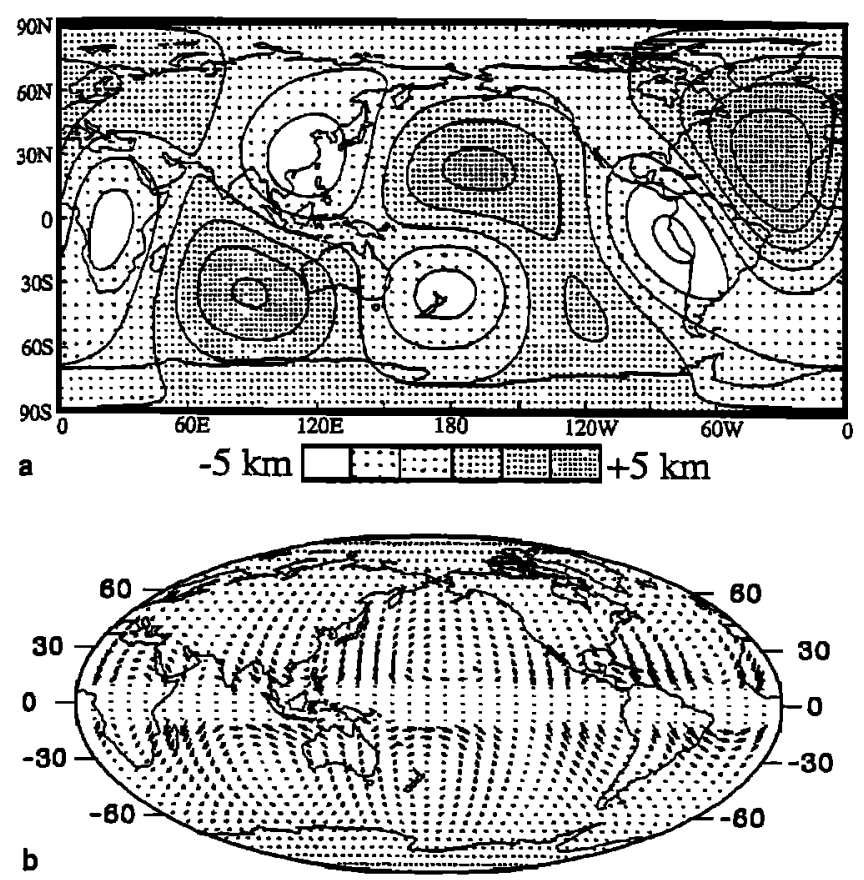

Fig. 2 a) Map of CMB topography obtained by Morelli and Dziewonski (1987). (Results are from inversion of compressional core phase data with spherical harmonic expansion up to degree and order 4.) b) Map of core fluid patterns based on the thermal wind model described in the text using results from part a).
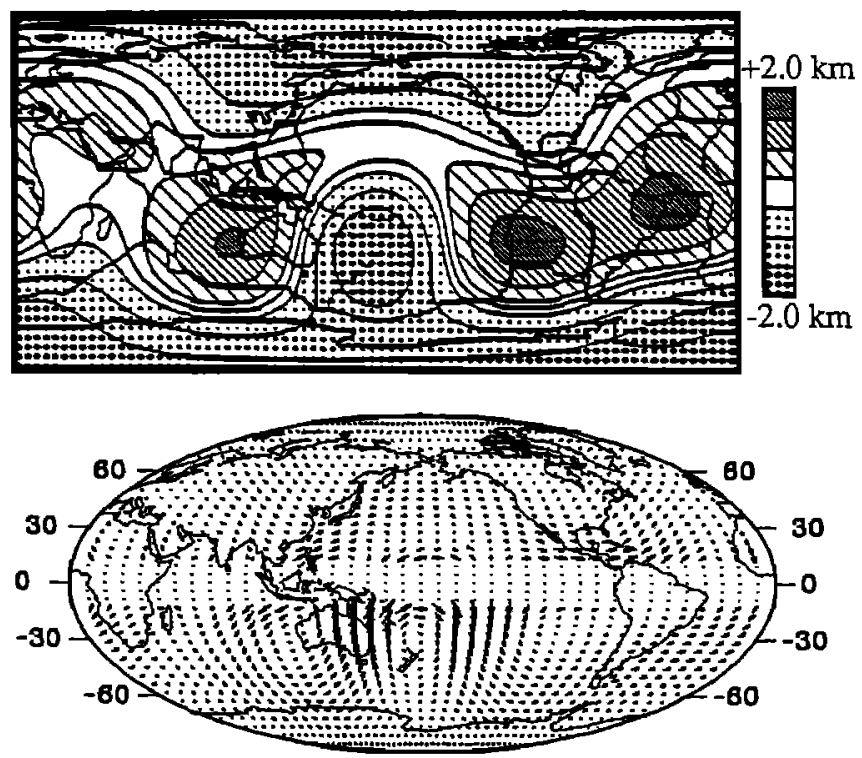

Fig 3. a) Map of CMB topography map obtained by Gudmundssen (1989), (Results are from inversion of compressional core phases with spherical harmonic expansion up to degree and order 5.) b) Map of core fluid patterns based on the thermal wind model described in the text using results from part a).

enormously different from values suggested by turbulent convection models [Stevenson, 1979, 1987], and we find $|\bar{v}|$ $\sim 10^{-2} \mathrm{~cm} / \mathrm{sec}$ comparable to westward drift. Clearly our model is most suited to estimating the pattern of core fluid and not the magnitude of that flow. For the same reason, uncertainties in the magnitude of $\mathrm{CMB}$ topography even by factors of ten are less important to assessing the model than the pattern of CMB topography.

\section{Discussion and Results}

Maps of the velocity field are given in Figures 2 and 3 with corresponding $\mathrm{CMB}$ topography maps. Figure $2 \mathrm{~b}$ shows the velocity flow field using the CMB results of Morelli \& Dziewonski (1987) and Figure 3b shows the velocity flow field using the results of Gudmundsson (1989). Both maps illustrate that closed loops of fluid flow are closely related to topography highs and lows, as expected. Although topography maps vary widely from worker to worker, there are features common to the maps studied here. For example, all maps show an anticyclone (due to the CMB topography high) underneath the Indian Ocean. Note that this flow is mostly but not purely toroidal. The continents have been drawn only for establishing the frame of reference.

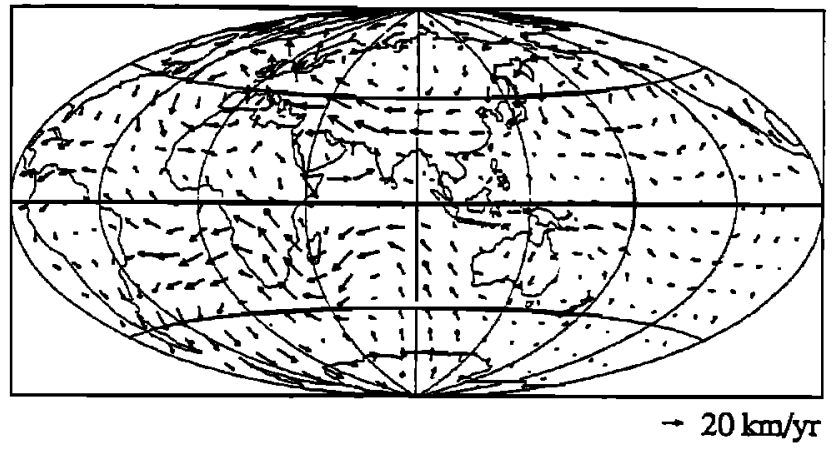

Fig 4. Core fluid velocity map of Bloxham (1989a) obtained from magnetic field data for time interval 1935-1940.

Comparisons with magnetic field data results of Bloxham (1989a) given in Figure 4, indicate that thermal winds may indeed be largely responsible for core fluid motions. The fluid flow map in Figure 4 is a result of magnetic field data during the time interval 1935-1940 but it displays similar general characteristics to maps of flow during other time intervals. For example, as in the velocity map given by thermal winds, fluid flow in Figure 4 is anticyclonic underneath the Indian Ocean and over south-central Asia. Our model is probably only relevant to field components that are steady although it is possible for dynamos to have steady velocity fields yet unsteady magnetic fields.

The expression for velocity containing the $l=2, m=0$ component of CMB topography expansion is given by

$$
\begin{aligned}
& v_{\theta}=0 \\
& v_{\phi}=-\frac{3}{2} \frac{g \alpha \varepsilon}{\Omega} \sin \theta g_{2}^{0}
\end{aligned}
$$

This expression describes the pattern of westward drift if the $1=2, m=0$ topography expansion coefficient is positive; fluid velocity is eastward if the coefficient is negative. It is the only term which globally describes this pattern. Figure $2 b$ shows strong westward drift in regions south of New Zealand and in the Indian Ocean. Figure 3b shows less westward drift and regions of strong eastward drift, possibly due to the large 
negative $\mathrm{l}=2, \mathrm{~m}=0 \mathrm{CMB}$ topography coefficient.

This model does not work near the equator since certain velocity terms diverge. This arises through our failure to retain $\theta$ and $\phi$ derivatives in the derivation of $v_{\theta}$ and $v_{\phi}$ (equations 3 and 4). More fundamentally, the Coriolis force cannot be expected to dominate near the equator and other effects, neglected here, will be important. However, the model behaves very well to within ten degrees of the equator and it can be used to predict patterns in core fluid velocity. In several respects, it is surprising that this model shows tentative evidence of validity. There are surely other sources of near-CMB flow and we have only determined one component. Moreover, the validity of the thin layer approximation $(\varepsilon<1)$ is debatable. Models that do not use this approximation (e.g., Bloxham, 1989b) show less correlation between flow and topography. As seismic and other models improve, it may become possible to decide whether a relatively thin layer thermal wind component contributes to core flow; we can thereby learn more about the core.

\section{Conclusions}

If outer core horizontal fluid flow is driven by horizontal temperature gradients due to $\mathrm{CMB}$ topography, then the thermal wind equations, expressed as a function of CMB topography, describe the advection of magnetic field resulting from core fluid motions. This model describes zonal drift by the velocity expression containing the $1=2, m=0$ topography coefficient and predicts that westward drift should not be a dominant feature in core fluid motions if CMB topography harmonic terms other than $1=2, m=0$ contribute much power. The thin layer version of our model predicts that anticyclones are associated with thermal and topographic highs, and a comparison of magnetically derived flow maps with our maps provides tentative support for this version.

Acknowledgments. We thank Jeremy Bloxham for discussions and Rob Clayton for the use of his computer graphics programs. This work is supported in part by NSF grant EAR-8816268. Contribution number 4872 of the Division of Geological and Planetary Sciences.

\section{References}

Bloxham, J., Simple models of fluid flow at the core surface derived from geomagnetic field models, Geophys. J. Int. 99, 173-182, 1989a.

Bloxham, J. On mapping the fluid flow at the core surface, Eos Trans. AGU, 70, 1075, 1989 b.

Bloxham, J. and D. Gubbins, The secular variation of earth's magnetic field, Nature, 317, 777-781, 1985.
Bloxham, J. and D. Gubbins, Thermal core-mantle interactions, Nature, 325, 511-513, 1987.

Flasar, F. M. and P. J. Gierasch, Turbulent convection within rapidly rotating superadiabatic fluids with horizontal temperature gradients, Geophys, Astrophys. Fluid Dyn., 10, 175-212, 1978.

Gubbins, D., Thermal core-mantle interactions and timeaveraged paleomagnetic field, I. geophys. Res. 23, 3413$3420,1988$.

Gubbins, D. and M. Richards, Coupling of the core dynamo and mantle: thermal or topographic? Geophys. Res. Lett. 13, 1521-1524, 1986.

Gudmundsson, O., Ph.D. dissertation, California Institute of Technology, 1989.

Hager, B. H. and R. W. Clayton, Constraints on the structure of mantle convection using seismic observations, flow models and the geoid, in Mantle Convection, edited by W. R. Peltier, Gordon and Breach, New York, pp. 657-763, 1989.

Hide, R., Interaction between the Earth's liquid core and solid mantle, Nature, 222, 1055-1056, 1969.

Jones, G. M., Thermal interaction of the core and the mantle and long-term behavior of the geomagnetic field, $\mathrm{I}$ geophys. Res., 82, 1703-1709, 1977.

Lay, T., Structure of the core-mantle transition zone, Eos Trans. AGU. 70, pp.49, 54-55, 58-59, 1989.

Merrill, R. T. and M. W. McElhinny, The Earth's magnetic field, 401 pp, Academic Press, New York, 1983.

Monin, A. S. and A. M. Yaglom, Statistical fluid mechanics. vol. 1, 769 pp., MIT Press, Cambridge, MA, 1971.

Morelli, A. and A. M. Dziewonski, Topography of the coremantle boundary and lateral homogeneity of the liquid core, Nature 325, 678-683, 1987.

Olson, $P$., Toroidal flow in the outer core and the thermal regime below the core-mantle boundary, Geophys. Res. Lett, 16, 613-616, 1989.

Pedlovsky, J., Geophysical fluid dynamics, 624 pp, Springer-Verlag, New York, 1979.

Stevenson, D.J., Limits on lateral density and velocity variations in the Earth's outer core, Geophys. J. Roy, astron. Soc., 88, 311-319, 1987.

Stevenson, D.J., Turbulent thermal convection in the presence of rotation and a magnetic field, Geophys. Astrophys. Fluid Dyn., 12, 139-169, 1979.

M. Kohler, Seismological Laboratory, California Institute of Technology, Pasadena, CA 91125

D. Stevenson, Division of Geological Sciences, California Institute of Technology, Pasadena, CA 91125

(Received: May 2, 1990; accepted: June 18, 1990.) 